\title{
Developing Geospatial Service-Oriented Architecture for Realizing Spatial Data Infrastructure
}

\author{
Behnam Tashayo \\ K.N. Toosi \\ University of Technology
}

\author{
Ali Mansourian \\ K.N. Toosi \\ University of Technology
}

\author{
M. Saadi Mesgari \\ K.N. Toosi \\ University of Technology
}

\begin{abstract}
Establishing the accessibility and usability of spatial data is one of the most important goals of $S D I^{I}$. In this respect, during recent years, a great number of spatial datalprocesses have been published through Open Geospatial Consortium $(O G C)$ interfaces. Due to absence of semantic interoperability, Users encounter many problems in finding and combining of OGC services. In this article, we propose a service oriented architecture to solve the existing problems in finding and combining of OGC services. In this architecture, semantic catalogue service and dynamic chaining were used for finding and combining of spatial services respectively. Furthermore, in the architecture, other OGC services were utilized for transformation, view and download spatial data. Semantic catalogue service generates and saves the ontology of submitted services automatically. Users can utilize semantic queries for accessing, invoking and interpretation of saved ontology. Therefore, the semantic web capabilities were used in the semantic catalogue service (without requiring any change in the publishing methods). In order to facilitate exchanging spatial data as the main goal of SDI, the catalogue service provides the possibility of exchanging information about spatial data in form of choreography. Finally, the proposed method was evaluated using a case study.
\end{abstract}

Keywords: Service Integration, Semantic Web, Dynamic Chaining, Choreography, Catalogue Service.

\section{Introduction}

Recent developments in web-based technologies (such as clearinghouse and catalogue service) facilitate presenting spatial data/process as service to achieve Spatial Data Infrastructure goals. Discovery, invoking and combining of spatial services require semantic and syntactic interoperability. Service integration involves all of these processes. Therefore,

\footnotetext{
${ }^{1}$ Spatial data infrastructure
}

Interoperability is more sensitive in service integration. In this respect, OGC service framework by establishing syntactic interoperability facilitates utilizing of spatial service; but due to lack of rules for understanding the content of these standards and specifications, there are still many problems in discovery, invoking and combining of OGC services. Considering widespread use of standard service [1], the mentioned problem is one of the main issues, prevents SDI goals.

Information society at a higher level, has offered various solutions to establish semantic interoperability. These solutions were used in several studies to establish interoperability in order to spatial service integration [3،2]. Usually, these studies have been concentrated on solving one specific problem. In addition, due to incompatibility of IT web service and OGC service framework [4] using the result of these studies require significant changes in publishing, discovery and invoking procedures of spatial services. These changes often comprise using IT standards or development of new components by service providers. Therefore, direct utilization of such solutions is not possible for spatial standard services.

In this study, a service-oriented architecture was designed with the aim of presenting solutions for semantic interoperability in spatial service integration. This architecture utilizes a semantic catalogue service to perform discovery process. The semantic catalogue service provides OGC services with semantic web capabilities, without modifying their publishing, discovery and invoking methods. This catalogue uses choreography to improve sharing of spatial data. Moreover, the proposed architecture uses semantic web capabilities in order to chain spatial services dynamically. Finally, we use a case study to investigate the proposed architecture capabilities.

\section{Research Background}

In this section, the idea of using service-oriented architecture in order to fulfill the requirement of spatial data infrastructure was discussed. For this 
purpose, primarily the concept of service integration was introduced. Subsequently, catalogue service and its capabilities were explained and finally semantic web and its facilities for spatial service-oriented architecture were investigated.

\subsection{Service integration}

Service user, service provider and service broker are main roles in service-oriented architecture. Service provider registers service in a metadata repository through a service broker (e.g., catalogue service or clearinghouse), User searches the desired service through the service broker and invoke it. Accordingly, the main roles of service-oriented architecture interact with publish, discovery and invoke processes.

The services can be combined in a service chain. Therefore, service combination was introduced as a new process in service-oriented architecture. Service combination in spatial field is one of the main advantages of SDI. This advantage generates great value-added for spatial services [5]. The services interact within a chain in form of orchestration or choreography. The purpose of orchestration is performing an executive process while choreography refers to transfer abstract messages among different parties. The most important difference between choreography and orchestration is having a central controller. In orchestration, a central controller keeps logic and controls activities. For instance, a user can orchestrate services such as conflagration history, temperature and pixels classifier to calculate fire risk in forest areas [6]. In choreography, abstract messages are exchanged between two or more parties and none of them can controls and manages the others. For example, a user visits a travel agency website and applies a ticket for a specified time, the travel agency connects to a number of transportation companies and informs them about the user request and receives their responses. The interaction between travel agency and transportation companies is in the form of choreography [7]. Different studies have been carried out on spatial service combination. These studies have focused on orchestration [9،8].

Service integration via service processes (discovery, invoking and combination) uses a set of distributed services (services that are often published by different providers) in order to solve a spatial issue. For this purpose, service integration requires syntactic and semantic interoperability. OGC standards only establish syntactic interoperability. Syntactic interoperability is sufficient when the meaning of various services and their input and output parameters are known. Therefore, service integration by means of syntactic interoperability obviates demands of some businesses. Nevertheless, in most cases, the complexity of spatial data and processes on the one hand and the multiplicity of spatial users and service providers on the other hand necessitate using of semantic description in service integration. In this study, we discussed integration of spatial services by establishing syntactic and semantic interoperability and using choreography and orchestration.

\subsection{Catalogue service}

The first step in integration of services is service discovery, which finds appropriate data/process. The importance of service discovery has led to present several standards by the information and spatial societies. For example, UDDI ${ }^{1}$ and ebRIM $^{2}$ were presented for $\mathrm{WSDL}^{3}$ base service and $\mathrm{CSW}^{4}$ was presented for spatial service. It should be mentioned that CSW is in fact a profile of ebRIM. In this standard, the interfaces, bindings and a framework for defining application profiles were specified to publish and access to spatial service metadata and descriptions.

In CSW, users can specify their desired criteria to search spatial services. However, only direct match of keywords regardless of their meanings is used for discovery. In other words, OGC standard does not support semantic search. For this reason, the capabilities of the mentioned standard are not adequate for complex spatial processes [2].

The efficiency of service discovery depends on descriptive information of the services. When various aspects of a service were well described, detailed queries are possible and sequentially responses will be more accurate. For this purpose, different studies have proposed using semantic web capabilities in order to improve discovery. In these studies, two main methods have been suggested. In the first method, catalogue service interface was modified in such a way that semantic queries can be performed. For example, Akkiraju et al. extended the Universal Discovery Description and Integration inquiry API to incorporate RDF expressions in the query [10]. In the second method, a middleware layer was used to set up semantic query capability. This layer matches queries and catalogue records. The second method was more treated in the literature [12،11]. As semantic catalogue service mature, data type, service type and finally process type ontologies with the aim of service chaining were developed [13]. These ontologies must be produced by providers, and then presented to users through catalogue service. In this paper, a semantic catalogue service was designed which used spatial process classification to facilitate dynamic service chaining. This catalogue produces standard service ontologies automatically and provides the possibility of using

\footnotetext{
${ }^{1}$ Universal Description, Discovery and Integration

2 electronic business Registry Information Model

${ }^{3}$ Web Service Description Language

${ }^{4}$ OpenGIS Catalogue Service
} 
descriptive information in service discovery. In the next section, the methods of presenting these descriptions by semantic web will be discussed.

\subsection{Semantic web}

The aim of Semantic Web is establishing a network that its contents will be machine understandable. To achieve semantic web, interpretation of contents should be legislated. Ontology provides required regulations. Ontology is an explicit and conventional specification that by defining the vocabularies and relationships between them describes a domain of knowledge. By using ontology, the meaning of exchanged content will be understandable for machine. Ontology in addition to semantic interoperability provides the possibility of reasoning and multipurpose usage of information [14].

Web Ontology Language (OWL) is a standard language for generating ontology. Expressiveness, flexibility, ease of use and universal acceptance are the advantages of this language. OWL-S is an OWLbased ontology for describing semantic web services. This ontology is used for facilitating service processes (including discovery, invoke and combination). The classes ServiceProfile, ServiceModel and ServiceGrounding constitute OWL-S. ServiceProfile includes provider information (e.g. contact information), service classification, functional, input and output descriptions and a section to provide any information about the service. ServiceModel describes the service preconditions and execution results. ServiceGrounding specifies access and execution details. Generally, an agent identifies the service with using ServiceProfile and utilizes it by means of ServiceModel and ServiceGrounding.

Different manual and automatic methods have been proposed to create ontology. Automatic methods often use natural language processing techniques to extract ontology from documents. Current automatic methods are not efficient. Therefore, manual or semi-automatic methods still are used to produce ontology [15]. In this study, an efficient method was proposed for automatic production of spatial web service ontology.

\section{Case study: designing a fiber optic network}

In a non-federated governmental system, different organizations participate in the management of city. Thus, service integration has great importance in the domain of urban management. In this section, designing a fiber optic network was discussed as a case study in this field.
In designing a fiber optic network, various technical and non-technical principles should be considered. Fiber optic network should be buried in the depth of $1.2 \mathrm{~m}$. the width of excavation must be at least $75 \mathrm{~cm}$ in order to allow inserting one container of cement and block to protect PVC pipes carrying fiber optic. Whatever the route of fiber optic will be closer to borders, maintenance and repair of the network will be easier. The distance of this network lines from other urban utilities such as water, sewage, gas and electricity should be considered $1 \mathrm{~m}$. Generally, the route of network should be designed in such a way that mechanical pressure, subsurface water, power stations effect and ... will be prevented.

Considering the above principles, designing the network requires street network and urban utilities dataset in addition to various spatial processes. In other words, the designer must integrate data and process, which were presented by various organizations. In the next sections, this case study was used in order to develop service-oriented architecture components.

\section{Semantic catalogue service}

Semantic catalog service establishes semantic interoperability in order to facilitate discovery of spatial services. An $\mathrm{XSLT}^{1}$ engine generates OGC service ontologies automatically. The catalogue service allows storage and usage of produced ontology or user desired ontology. In addition, the semantic catalogue utilizes a choreography processor to request confirmation about data, data inquiry and generally exchanging message about spatial data between users and service providers.

\subsection{Motivation of a semantic catalogue service}

In this section, with the help of the case study, the necessity of using semantic catalogue service in service integration was investigated. The first phase of designing fiber optic network includes finding required data and processes through a discovery service. For this purpose, the designer uses a catalogue service, which constructed based on spatial data infrastructure. Current catalogue services search using keywords (e.g. water network). The responsible organizations of urban utilities (such as water, electricity ...) mostly use different vocabulary to name the same feature. Therefore, comprehensive results often cannot be achieved by searching the features with their name. If the features are searched with the name of responsible organization, all data that related to that organization will be retrieved, but

$\overline{1}$ extensible Style Language Transformation 
still there are many problems for using such data. For example using different vocabulary will encounters the user with difficulty in understanding the quiddity of data. In addition, the considerations of other utilities will not be presented to the user (e.g. gas lines are required to keep 1.5 meters away from other utilities). While the user needs to know the considerations of other utilities in order to perform the design.

Semantic interoperability solves the mentioned problems. To establish semantic interoperability, semantic descriptions of spatial service must be present to user. If the semantic descriptions of spatial services will be presented by catalogue service (as service broker), the users and providers who communicate through the catalogue can interact semantically.

The second phase of designing fiber optic network is using the gathered data to route network lines. Various urban utilities should be constructed in a limited space under the ground. The coordination among organizations for using this common space prevents future problems in the development and maintenance of infrastructures [16]. In this respect, it is essential to establish cross-organizational communication between the responsible organizations. SDI guidelines necessitate all urban organizations to use catalogue service. Consequently, this service is convenient to set up crossorganizational communication in order to exchange messages about spatial data. By establishing such communication, whenever the user cannot find the desired data even by means of semantic descriptions, the catalogue service can be used to send requests to various organizations. In addition, by considering the importance of developing infrastructure and usually according to the existing criteria in urban management, cross-organizational communication enables users to inquire the accuracy and completeness of registered data from responsible organizations.

\subsection{Methodology}

According to the analysis of case study in section 4.1 , various characteristics should be considered in the designing of a semantic catalogue service:

- In order to establish semantic interoperability for OGC services, the catalogue service must produce the ontology of such services automatically.

- The catalogue service should provide the possibility of storage and retrieval of produced ontology or other users' desired ontology and semantic descriptions.

- The catalogue service should provide the possibility of inquiry or obtaining confirmation from the data provider organizations.
- The catalogue service should answer to thematic and spatial queries (according to the OGC standard) furthermore to semantic queries.

CSW supports developing new profiles to facilitate publishing and discovery of spatial services. Stock et al., by developing CSW interface have presented a profile with the ability of response to semantic queries [17]. The semantic capabilities of this profile had been improved by supporting Sparql language and adding some parameters to service operations while all the main capabilities of CSW interface were preserved. This profile was used as the initial core of semantic catalogue service. The profile Interface supported OWL DL language, while for describing OWL-S ontology, OWL FULL language was needed which offers more details than OWL DL. In this study, the service interface was extended to support OWL FULL and by adding three more components, the required characteristics of semantic catalogue service were provided. These components includes $\mathrm{XSLT}^{1}$ engine for automatic production of ontology, semantic processor for storage, display, load, edit and query of OWL-based ontology and choreography processor to exchange messages about spatial data (Fig. 1). Each of these components will be more described in the following.

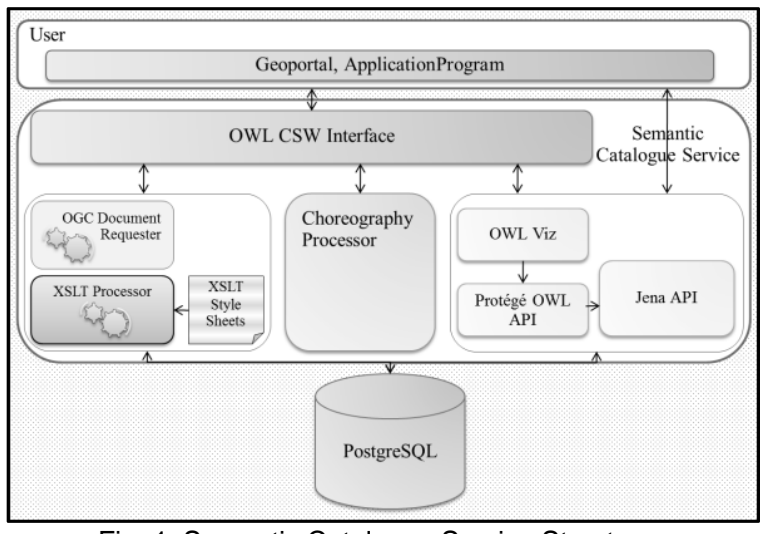

Fig. 1: Semantic Catalogue Service Structure

4.2.1. XSLT engine. This component includes a repository of XSLT style sheet, an XSLT processor and an applicator to request OGC documents. As mentioned in section 2.3, service ontology (OWL-S) is constructed via three classes. The Information presented by the classes is similar to the existing information in the messages of OGC services (e.g. GetCapabilities). In other words, the information that is required to produce the ontology of spatial service is currently presented by OGC services but it is not usable by semantic web tools. The OGC services messages are XML-based and similar for one service type. For example, all WPS services present

\footnotetext{
eXtensible Style Language Transformation (XSLT is an XMLbased language that describes the transformation of XML file into other files (e.g. OWL, HTML))
} 
GetCapabilities messages with the same structure. Therefore, we can establish a correspondence among the messages of OGC services and OWL-S classes and produce XSLT style sheets for each of these services. The style sheets will be similar for all instances of one service type. The applicator retrieves documents according to the type of registered services. Then, the XSLT processor utilizes these documents together with stored style sheets to generate the ontology of services automatically.

In this paper, by establishing correspondence between WFS and WPS messages and OWL-S classes, their style sheets were produced. In addition to OGC documents, other entries proportionate to service type can be used to produce style sheets. This method improves the semantic descriptions and facilitates the service tasks. In this regard, the information of processing service classification (according to ISO 19125) were stored in a WPS profile, this profile is used along with GetCapabilities and DescribeProcess documents to generate OWL-S classes for WPS services (Fig. 2).

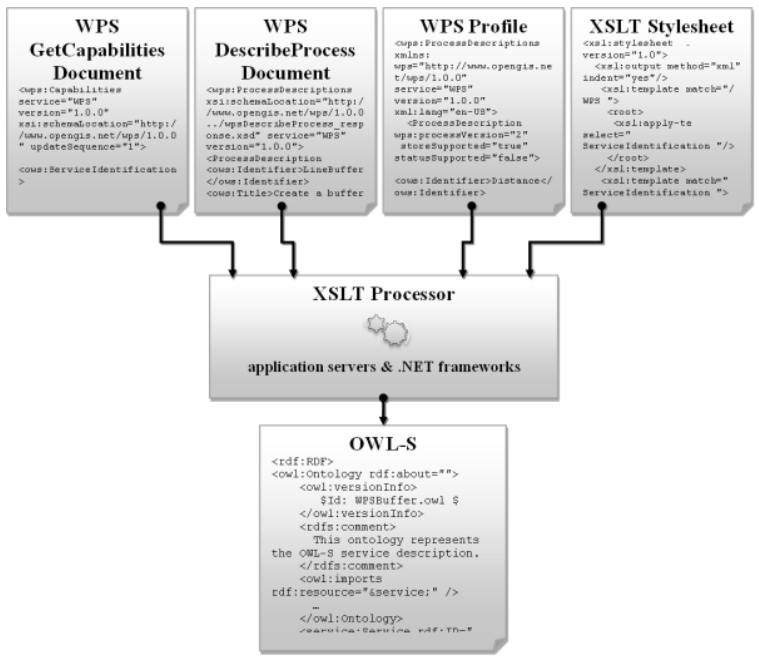

Fig. 2: The procedure of Ontology generation for WPSs

4.2.2. Semantic processor. The interface of semantic catalog service should provide the possibility of storage, load, display, edition, and query of OWL-based ontologies. The semantic processor was used to create the mentioned capabilities in the catalogue service. OWL Viz, Protégé OWL API and Jena API were used in development of this processor. OWL Viz was used for display and visual editing of OWL-based ontology. In order to use the capabilities of this editor, Protégé OWL API ${ }^{1}$ was required. This API also depends on Jena API. Moreover, The Jena API is responsible for analysis of semantic queries, inference and communication with the database. The relationship between the semantic processor and

${ }^{1}$ Application program interface other components of semantic catalogue service is shown in Fig. 1. This method of using semantic web capabilities solves direct incompatibility of IT technologies and OGC services frameworks in service discovery.

4.2.3. Choreography Processor. Urban management requires exchanging message about spatial data in order to efficient spatial data sharing between responsible organizations (Section 4.1). Prediction of all issues in which exchange of messages becomes necessary is not possible. The establishment of static communication among various organizations with considering the dynamic nature of required communications is not efficient. The information society has proposed the concept of choreography to provide a solution for dynamic cross-organizational communication. In this section, a choreography method was proposed in order to exchange messages about spatial data by means of semantic catalogue service.

Automating the communication among organizations requires an agreement about the structure and content of messages. Usually XMLbased documents with conventional structure have been used as messages [18]. In this study, Filter Encoding standard was used to develop the structure of XML-based messages. Filter standard provides Ad hoc XML documents, which can be used for one specific communication. Due to unpredictable communications, production of Ad hoc documents is necessary for choreography. Encoding of this standard is independent from platform and XML tools can analyze or even convert it to another query language (such as SQL). In addition, Various OGC standards use Filter. For example, all of catalogue services, which are developed based on CSW standard is constrained to support Filter standard. Thus, many popular spatial servers support this standard. Consequently, spatial servers of various organizations require a negligible development to support choreography of messages produced by means of Filter (Fig. 3).

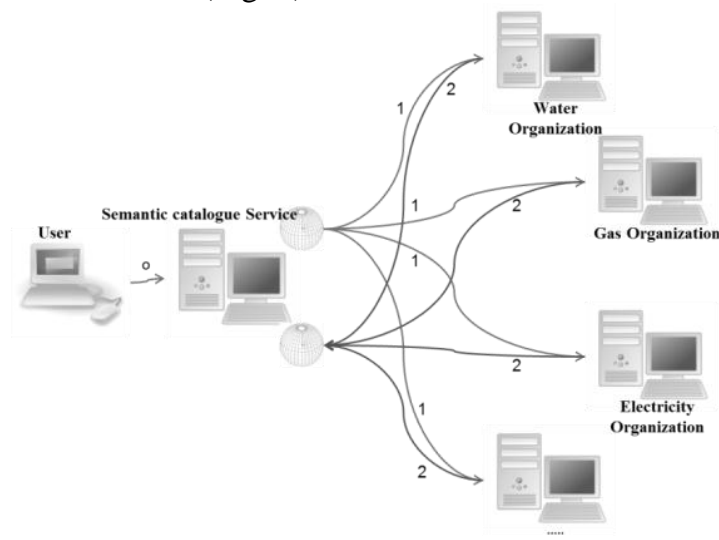

Fig. 3: Message transformation with using choreography processor 
The structure of Filter-based messages only includes XML elements. Therefore, each message can be considered as a string. This string should be encoded based on Key Value Pair (KVP) and transferred using HTTP GET method. The user sends a request to catalogue service in Filter format. The catalogue service analyzes the message, according to the content sends it to relevant organizations, and then sends back their answers to the user. In section 5 , the user interface operation that facilitates exchanging of messages will be discussed.

\section{Design and development of a prototype system}

In order to evaluate the proposed methodology in service integration, a prototype service-oriented architecture was developed. In the designing of a service-oriented architecture, the required capabilities should be shared among various services in such a way that maximizes the multi-purpose capability, performance, flexibility and usability of them. For example, if a service provides complicated process that compromise several actions, due to fewer service invocation the performance may increase but reusability of the service in different scenarios may decrease.

In this paper, the proposed architecture was designed according to INSPIRE guidelines (Fig. 4). INSPIRE-based architectures have several advantages, which enhance data and service interoperability [19]. INSPIRE classifies all of the required services into five categories: invoke, transformation, download, view and discovery.

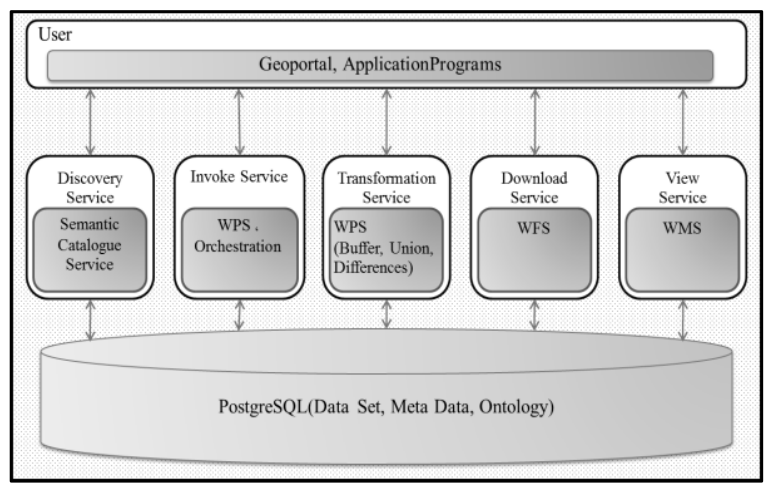

Fig. 4: The Proposed Service-Oriented architecture

According to INSPIRE, The "Invoke Spatial Data" service supports invoking individual (spatial) services as well as combinations of individual (spatial) services in service chains through a (web) service orchestration engine. This service enables a user to run spatial services without requiring the availability of a GIS. In the proposed architecture, WPS was used as coordinator in orchestration process.
Transformation service converts input data to user's desired data. In other words, this service provides required process. In order to provide the required processes in the case study, three processes; buffer, union and difference were implemented as WPS standard services. Furthermore, WFS interface was used as download service, WMS was used to display data in user interface and semantic catalogue service is responsible for service discovery.

We consider two hypothetical users in order to investigate the proposed system. The first user is a service provider who wants to register a service in the catalogue and the second user is a fiber optic network planner. The first user primarily selects the service type (e.g. WFS, WPS, and ...) through interface, and if the service was a WPS therefore specifies its process type. In Fig. (5), the user is registering a buffer service in the catalogue. Moreover, according to OGC specification, the user can update and remove service from catalogue service.

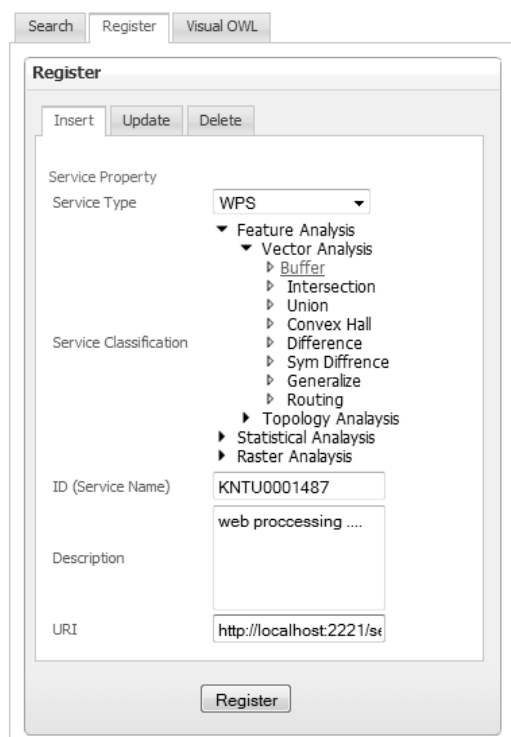

Fig. 5: service registration in semantic catalogue service

The planner can search ontology in the knowledge base or invokes his desired ontology through geoportal interface. He inputs the desired ontology address (an OWL-based ontology developed for coordinating the design of co-located urban infrastructure) into the user interface; the mentioned ontology is loaded and displayed. By means of this ontology, the planner specifies all datasets (such as pipelines, power distribution stations and...) that should be considered in designing of fiber optic network. Then by using the interface, he searches all datasets in the extent of the plan (Fig. 6) and among the found datasets chooses corresponding datasets with the ontology specified datasets. 


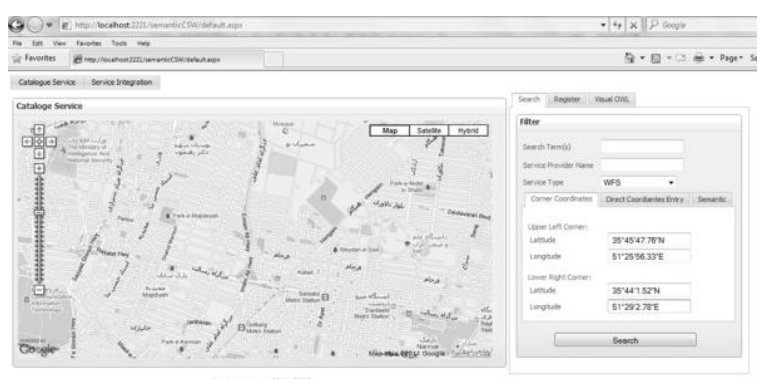

Fig. 6: 1st step search, Spatial search in user desired Boundary

Some of the required datasets maybe do not be among the found datasets or had saved with an unaccustomed name to the user. For example, wastewater wells must not located on the route of fiber optic network, but there is no dataset with this title in found datasets.

The next step for more accurate results is using semantic query. Planner by providing details such as owner and data type performs a semantic query through the user interface using Sparql language but the semantic search has no result too (Fig. 7).

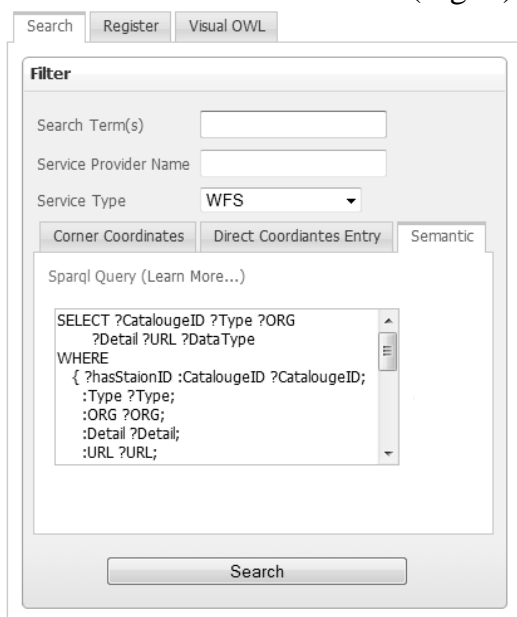

Fig. 7: 2nd Step Search, Using Semantic Query

The next step is search using choreography. In this method, the user sends a message in Filter language to semantic catalogue service in order to request the desired dataset (Fig. 8). The catalogue service analyzes the received message and forwards it to relevant organizations. If the requested dataset was available in these organizations, the user will be informed.

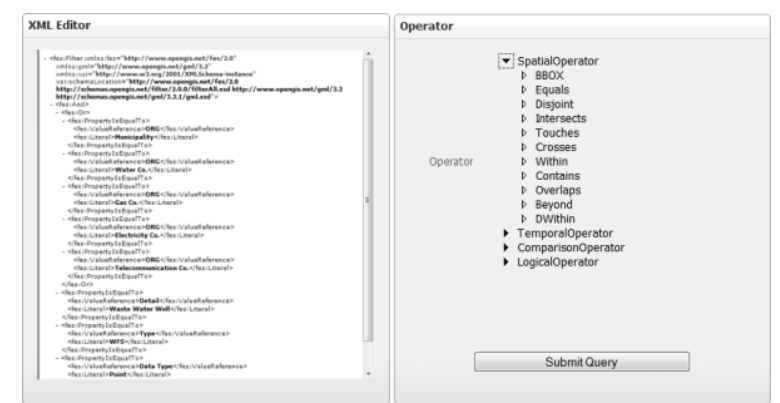

Fig. 8: 3th step in Search, using choreography messages
By using semantic catalogue service and with the help of ontology, in addition to the desired datasets, the essential constraints and considerations of the datasets are also accessible. For example, the abovementioned ontology includes two types of micro and macro constraints for route selection to implement facility lines (Fig. 9). Macro constraints include surrounding issues of fiber optic network such as residential, business and traffic impact, maintenance and networking issues and its conflict with other urban facilities. Each of these constraints concerns multiple subjects, for example, networking concerns excavation depth, work zone width and soil type. Micro constraints indicate spatial constraints for fiber optic network such as clearance constraint. According to the aim of the article, only spatial constraints were regarded in designing of network.

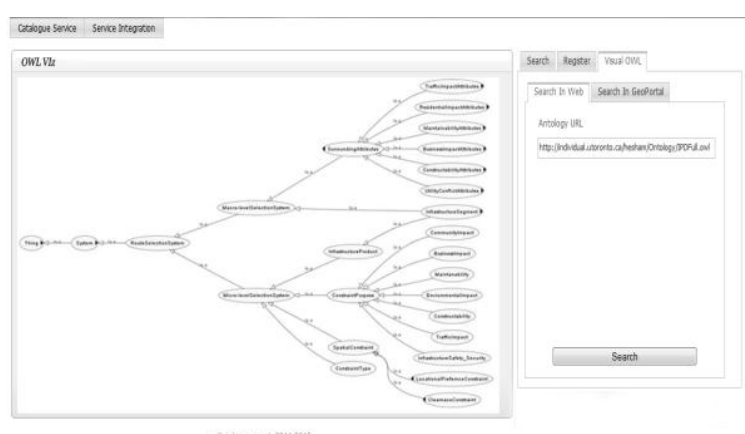

Fig. 9: show user desired Ontology in Geoportal Interface

According to ontology, the clearance distance for water, electricity, telecommunication and fiber optic lines is $1 \mathrm{~m}$, for gas lines is $1.5 \mathrm{~m}$ and for wastewater network is $.5 \mathrm{~m}$. Moreover, the distance of fiber optic lines from power distribution stations and wastewater wells should be $1.5 \mathrm{~m}$.

The user by utilizing the collected information (e.g. utilities requirements and road network dataset), performs the required processing to determine the area that can be used for implementation of fiber optic network. For this purpose, the invoke service will be used (Fig. 10).

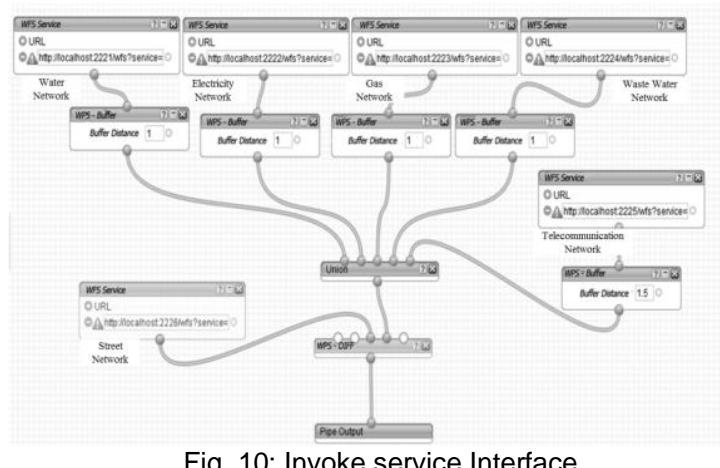

Fig. 10: Invoke service Interface

At first, the planner specifies the clearance distance of other urban facilities according to their types by using a buffer processor. Then all of the clearance distances are merged and their difference 
with pavement is calculated. The remaining area can be used for implementation of fiber optic network.

The invoke service considers several services (from processing services that registered in the catalogue) for each of the processes in the service chain. For example, if one of the required processes in the chain is buffering, several buffer services will be assign to that node in the chain. In this manner if one service cannot respond, it will be replaced with another service dynamically.

\section{Discussion and Conclusion}

In recent years, there have been great advances in publishing spatial data and process through OGC standards in order to develop SDI. Mostly, the required information is not directly provided by spatial services. Therefore, users should generate the required information by means of existing data and processes. The first step to utilize existing service is finding the appropriate data/process service. Discovery of suitable service is dependent on service descriptions. Whatever, service properties were described with more details, precise queries and proper responses are potentially possible. Semantic web by providing semantic descriptions for web service improves its discovery procedure and set up a suitable foundation for optimal using of available services.

Catalogue service as a broker between user and service provider is an appropriate tool for providing semantic descriptions. Various studies have been carried out to improve semantic interoperability by using brokers but these studies are mainly limited to storage of semantic descriptions in these brokers. In this study, a semantic catalogue service was developed which produces and stores the ontology of spatial service automatically. This service by supporting semantic query provides the possibility of accessing, invoking and interpreting of the stored ontology. In addition, the catalogue user interface can be used to display and modify the ontology. Considering the mentioned characteristics, the semantic catalog service has provided semantic web capabilities in service discovery without any changes in publishing method of OGC services. Simultaneous support of semantic and spatial query has turned the catalogue service to an interface for establishing connection between IT technologies and OGC services framework.

In order to establish an environment to facilitate the sharing of spatial data as the main goal of SDI, the exchange of message about spatial data is necessary. According to broker role of catalogue service, in this study it was used to exchange messages in choreography manner.

In this paper, a service-oriented architecture was developed based on INSPIRE guidelines which benefits from semantic catalogue service as the discovery service. In the proposed architecture, the dynamic combination of services was provided. This means that whenever a service cannot be served, an alternative service will be substituted automatically. The operation of the mentioned architecture in integrating spatial service was discussed using a case study.

The ontology of different services was used to improve service discovery but for service chaining as an essential stage of service integration various chaining technology such as WPS and BPEL were used independent of ontology yet. Incorporation of semantic descriptions and service chaining technologies improve service integration and automate the chaining process completely. The authors in their future studies will investigate the possibility of incorporating semantic descriptions and service-chaining technology within spatial domain.

\section{References}

[1] Wu, H. Li, Z. Zhang, H. Yang, C. and Shen, S. (2010) Monitoring and evaluating the quality of web map service resources for optimizing map composition over the internet to support decision making. Computers and Geosciences, (in press).

[2] Yue, P. Di, L. Yang, W. Yu, G. and Zhao, P. (2007) Semantics-based automatic composition of geospatial web service chains. Computers and Geosciences, Volume 33(5), pp. 649-665.

[3] Lacasta, J. Nogueras-Iso, J. Bejar, R. Muro-Medrano P.R. and Zarazaga-Soria, F.J. (2007) A web ontology service to facilitate interoperability within a spatial data infrastructure: applicability to discovery. Data and Knowledge Engineering, Volume 63 (3), pp. 947-971.

[4] Sample, J. Shaw, K. Tu, S. and Abdelguerfi, M. (2008) Geospatial services and applications for the Internet. Springer Science Business Media, LLC, 233 Spring Street, New York, NY 10013, USA.

[5] Einspanier, U. Lutz, M. Senkler, K. Simonis, I. and Sliwinski, A. (2003) Toward a Process Model for GI Service Composition. Munster, Germany: Institute for Geoinformatics, University of Munster. pp. 31-46.

[6] Friis-Christensen, A. Lucchi, R. Lutz, M. and Ostlander, N. (2009) Service chaining architectures for applications implementing distributed geographic information processing. International Journal of Geographical Information Science, Volume 23(5), pp. 561-580.

[7] Peltz, P. (2003) Web services orchestration and choreography. IEEE Computer, Volume 36(10), pp. 4652.

[8] Kiehle, C. Greve, K. and Heier, C. (2007) Requirements for next generation spatial data Infrastructures-standardized web based geoprocessing and web service orchestration. Transactions in GIS, Volume 11(6), pp. 819-834.

[9] Lemmens, R. Granell, C. Wytzisk, A. de By, R. Gould, M. and van Oosterom, P. (2006) Integrating semantic and syntactic descriptions to chain geographic services. IEEE Internet Computing, Volume 10(5), pp. 42-52.

[10] Akkiraju R, Goodwin R, Doshi P, Roeder S (2003) A 
method for semantically enhancing the service discovery capabilities of UDDI. In: Proceedings of the Workshop on Information Integration on the Web, Eighteenth International Joint Conference on Artificial Intelligence (IJCAI), Mexico, pp. 87-92.

[11] Yue P, Di L, Zhao P, Yang W, Yu G, Wei Y (2006) Semantic augmentations for geospatial catalogue service. In: Proceedings of the 2006 IEEE International Geoscience and Remote Sensing Symposium (IGARSS06), 31 July-4 August 2006, Denver, USA. pp. 3486-3489.

[12] Srinivasan N, Paolucci M, Sycara K (2004) Adding OWL-S to UDDI, implementation and throughput. In: Proceedings of First International Workshop on Semantic Web Services and Web Process Composition, San Diego, USA 2004. 12 pp.

[13] Yue P, Gong J, Di L, He L, Wei Y (2011) Integrating semantic web technologies and geospatial catalog services for geospatial information discovery and processing in cyberinfrastructure. GeoInformatica, Volume 15(1), pp. 273-303.

[14] Zhao, P. Di, L. Yang, W. Yu, G. and Yue, P. (2008) Geospatial Semantic Web: Critical Issues. In Karimi, H.A. (Ed.) Handbook of Research on Geoinformatics. Information Science Reference, New York.

[15] Laender, A. Ribeiro-Neto, B.A. da Silva, A.S. and Teixeira, J.S. (2002) A brief survey of Web data extraction tools. ACM Sigmod Record, Volume 31(2), pp. 84-93.

[16] Novick, D. (1990) Life-Cycle Considerations in Urban Infrastructure Engineering. Journal of Management in Engineering, Volume 6(2), pp. 186-196.

[17] Stock, K. Small, M. Ou, Y. and Reitsma, F. (2009) OWL Application Profile of CSW. OGC Discussion Paper, Technical report, Open Geospatial Consortium.

[18] Arroyo, S. Duke, A. Lopez-Cobo, J. and Sicilia, M. (2007) A model-driven choreography conceptual framework. Computers standards and Interfaces, Volume 29(3), pp. 325-334.

[19] Granell, C. Diaz, L. and Gould, M. (2010) Serviceoriented applications for environmental models: Reusable geospatial services. Environmental Modeling and Software, Volume 25(2), pp. 182-198 